\title{
APPLYING CLOUD COMPUTING TECHNOLOGY TO BIM VISUALIZATION AND MANIPULATION
}

\author{
Tien-Hsiang Chuang ${ }^{1}$, Bo-Cing Lee ${ }^{1}$, and I-Chen $\mathrm{Wu}^{1 *}$ \\ ${ }^{1}$ Department of Civil Engineering, National Kaohsiung University of Applied Sciences, Kaohsiung, Taiwan \\ *Corresponding author (kwu@cc.kuas.edu.tw)
}

\begin{abstract}
A current trend in the construction sector is the application of Building Information Modeling (BIM) for the efficient integration and management of engineering information. Today, engineering consultant, construction and architectural companies conduct research into the development of BIM in order to integrate and visualize engineering information. The capabilities of BIM for information integration, visualization and parametric design, reduce duplication of work and interface integration complexity, significantly saving both time and cost. At present, BIM desktop applications such as Autodesk Architecture, Bentley Architecture and Graphsoft ArchiCAD, can only integrate and visualize information on-site. Difficulties remain in obtaining and updating information from other sites, which restricts communication and information distribution between different sites. As visualization efficiencies and hardware capabilities mature and continue to improve, the development framework of applications is now moving form "Client-Server" to "Host-based", which is referred to as "Cloud Computing” technology. In this research, we utilize the concept of Software as a Service (SaaS) and Cloud Computing in order to develop a visual system for BIM visualization and manipulation. This system can not only visualize three-dimensional (3D) BIM models, it can also manipulate 3D BIM models through the web without the limitations of time or distance. This system can facilitate communication and distribution of information between related participants, such as construction companies, building owners and architectural companies in order to manage projects effectively and efficiently.
\end{abstract}

Keywords: BIM, Cloud Computing, SaaS, Information Visualization, Information Manipulation

\section{INTRODUCTION}

The phrase "Building Information Modeling" (BIM) was coined in early 2002 by industry analyst Jerry Laiserin to describe virtual design, construction, and facilities management. BIM processes revolve around virtual models that make it possible to share information throughout the entire building industry [1]. Moreover, BIM has been in the focus of Research in Computer Aided Engineering for many years. A large number of publications have been written covering an extremely broad range of topics from fundamental technologies to practical implications. Commercial BIM systems, such as Autodesk Revit, Bentley Architecture and Tekla Structures, were developed specifically for integrating and visualizing project information. Because these systems are based on standalone system frameworks, it is difficult for participants from different sites to access a common set of information. As a result, BIM users are restricted in terms of communication and information distribution. In this research, we propose the concept of a Cloud-BIM system. This concept moves the BIM visualization and computing framework form "Single-alone" to "Host-based" - "Cloud Computing” technology as shown in Figure 1. We utilize the concept of Software as a Service (SaaS) of Cloud Computing [2] to develop an interactive system for BIM visualization and manipulation. This system can not only visualize and manipulate BIM models through the web without time and distance limitations, it also provides easyto-use web services for the various project participants, including construction companies, business owners and architectural companies, to access and view project information effectively and efficiently via the web. 


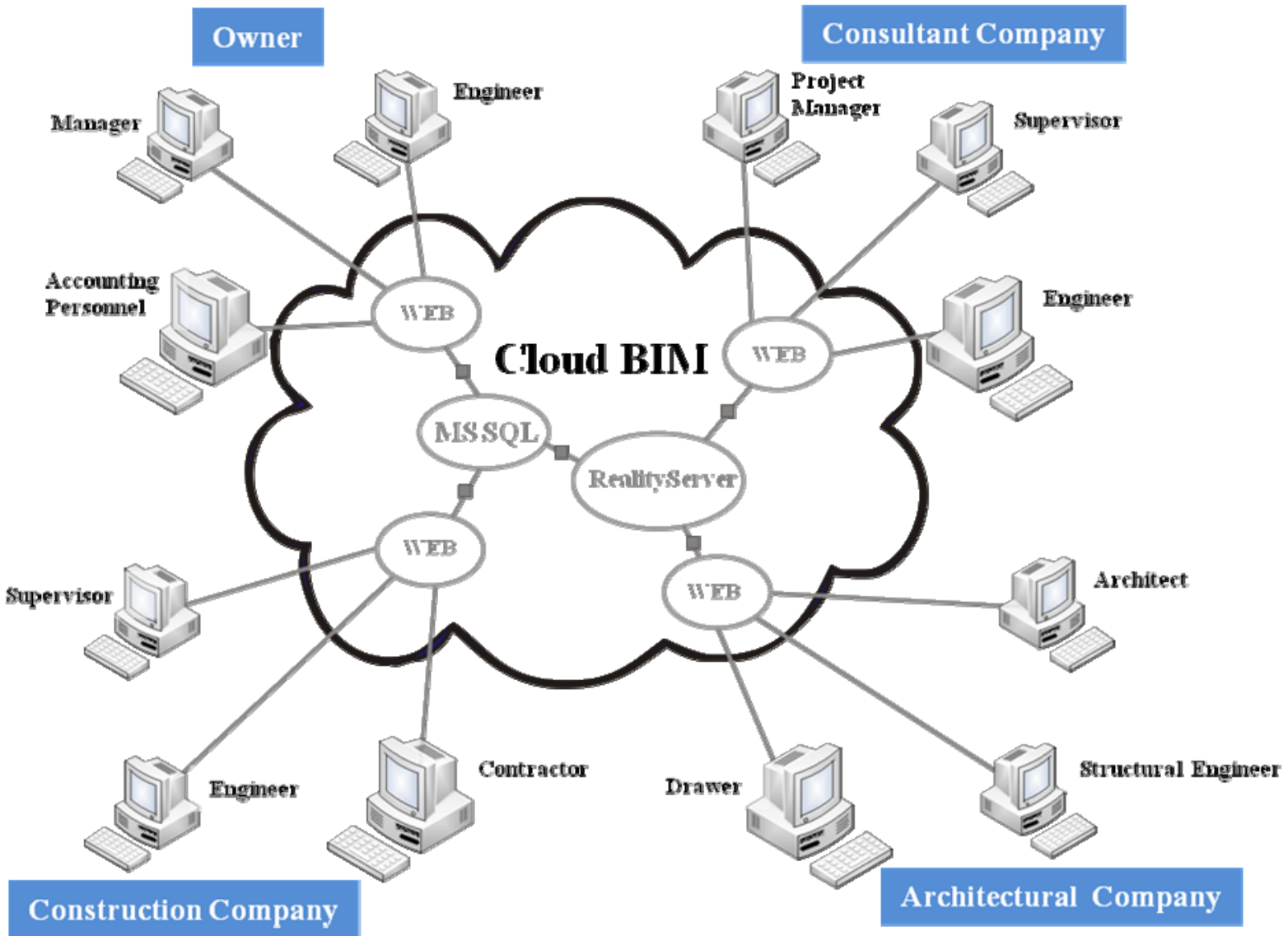

Figure 1. The concept of Cloud-BIM

\section{CLOUD COMPUTING TECHNOLOGY}

Currently, general BIM systems, being site-specific, limit the use of data visualization and manipulation by users located at different sites. In order to address this issue, this research utilizes the concept of SaaS with regards to Cloud Computing, which is a software delivery method that provides access to the software and its functions as a webbased service, to develop a visual system for BIM visualization and manipulation. "Cloud Computing” is a technology used to access services offered in the Internet cloud. Everything an informatics system has to offer is provided as a service, so users can access these services available in the "Internet cloud" without needing to have any previous know-how on managing the required computing resources. The Cloud-BIM system not only provides an information visualization service, it also provides information manipulation services for users located anywhere, and at any time.

\section{BIM VISUALIZATION}

Through a large number of experiments, Treicher [3] has proven that $83 \%$ of the information received by humans is by the sense of sight, supporting the view that information visualization is most essential for communication and information distribution. Therefore, an effective project management system should not only provide sufficient and comprehensive information to facilitate project management, it should also provide the various visualization tools to assist with information distribution and communication. To meet these needs, a Cloud-BIM system provides 1D (text), 2D (graph and chart), 3D (3D Model) visualization for ease of communication among the project participants. The Cloud-BIM system is able to present significant information in multiple modes such as text and graphical formats, by accessing the database of the Cloud-BIM system. For 3D Visualization, the Cloud-BIM system employs RealityServer [4, 5] as the visualization engine for BIM. RealityServer is the visual web computing 
platform for developing and deploying internet-based 3D applications and services. RealityServer conforms to the concept of cloud computing, which uses the server for application state and rendering, instead of placing the resource burden on the client. Real Internet-based 3D application services are also referred to as 3D Web Services. This architecture provides a flexible framework for handling incoming client requests, manipulating the user state, managing rendering operations, and transmitting information back to the client. The client communicates with the server through the web using HTTP. Images are rendered sequentially, pixel by pixel, in a sweeping vertical or horizontal motion, and suitably compressed images are delivered to the client, as shown in Figure 2. Therefore, image quality in the final stage will be higher than that in the early stage.

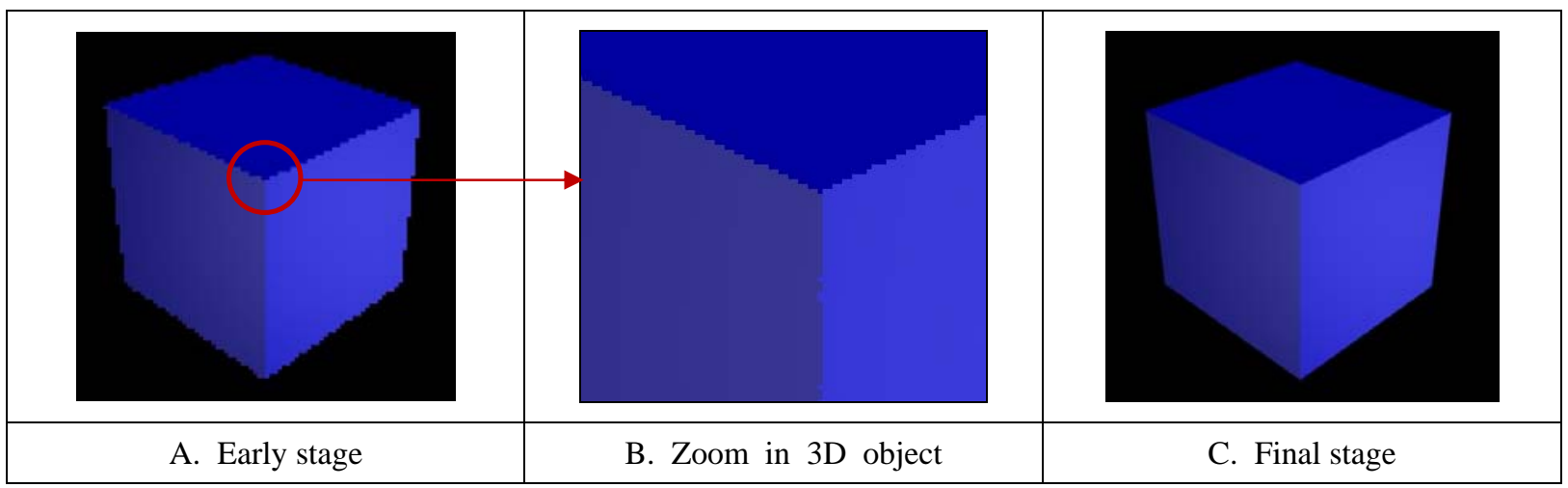

Figure 2. The principle of rendering image in RealityServer

\section{BIM MANIPULATION}

This research designed and implemented an interactive Graphical User Interface (GUI) to improve BIM visualization and manipulation to assist people in efficiently acquiring applicable information through the web. A GUI can be defined as an interface that allows humans to interact with computers using visual indicators in conjunction with text, a control selector (usually a mouse) and a screen. Creating websites is easy, but creating sites that truly meet the needs and expectations of the wide range of possible online users can be quite difficult. Designers need to base their work on the principles of design; interfaces have to be intuitive, clear, fast, pleasurable in use, and meet psychological demands [6]. In recent years, there has been a renewal of interest in how to design effective user interfaces for applications. Researchers such as Shneiderman et al. propose eight golden rules of user interface design [7]; Jenifer Tidwell captured UI best practices and reusable ideas as design patterns for facilitating UI design [8]; Fowler et al. developed a guidebook to design good UIs for web applications [9]; Jakob Nielsen and Hoa Loranger shared their insightful thoughts on web usability for webpage, content, site, and intranet design [10]. The ISO 9241171:2008 [11] also provides ergonomic guidance and specifications for the design of accessible software for use at work, in the home, in educational institutions and in public places. Synthesis of the aforementioned work has enabled us to distill the most important application design principles for our work:

- Visibility: The system should always keep users informed about what is occurring.

- Feedback: The system should respond effectively to a query or outcome.

- Ease of remembering: It should be easy to remember how to use the functions in the system.

- Mapping: The system should follow real-world conventions and make information appear in a natural and logical order.

- Consistency: Users become accustomed to certain actions and sequences, and they are likely to become confused when they encounter unexpected behavior.

Thus, this research designed and implemented an effective UI based on the abovementioned principles of design, 
which was carried out in the Microsoft .Net environment using Silverlight for Rich Internet Application (RIA), to enable users to access and visualize information through Web applications.

\section{SYSTEM DESIGN AND IMPLEMENTATION}

\subsection{SYSTEM FRAMEWORK}

This research developed the Cloud-BIM system which can facilitate BIM visualization and manipulation for project control and monitoring. The implementation of the CloudBIM system was carried out in the Microsoft Visual Basic .NET environment and provides web-based services, including BIM visualization and manipulation for facilitating efficient and effective interaction with the system. As Figure 3 shows, the Cloud-BIM system can be divided into three tiers: Client-Side Presentation Layer, Enterprise Application Service Layer and The Integrated Data Layer. Further details will be discussed in the following section.

\section{Tier 1: Client-Side Presentation Layer}

An effective client UI was designed and implemented in the Microsoft .Net environment using Silverlight for RIA, for information access and visualization through web applications.

\section{Tier 2: Enterprise Application Service Layer}

Tier 2 is primarily geared towards providing Enterprise Application Services via web services based on the concept of SaaS. The Cloud-BIM system can be deployed on the server side of the internet using Cloud Computing services, or on-site for use in and across the enterprise. In this research, the Cloud-BIM system adapted the concept of SaaS as the key software delivery method. It provides access to the software and its various functions as a webbased service. SaaS allows the entire set of project participants to access business functionality at a lower cost, without heavy technology implementation requirements.

\section{Tier 3: The Integrated Data Layer}

Tier 3 comprises the Integrated Data Layer which is responsible for describing and storing the entire set of information about the BIM. The relational database management system, MS SQL Server from Microsoft is used to interact with the database which stores all the information about the BIM.

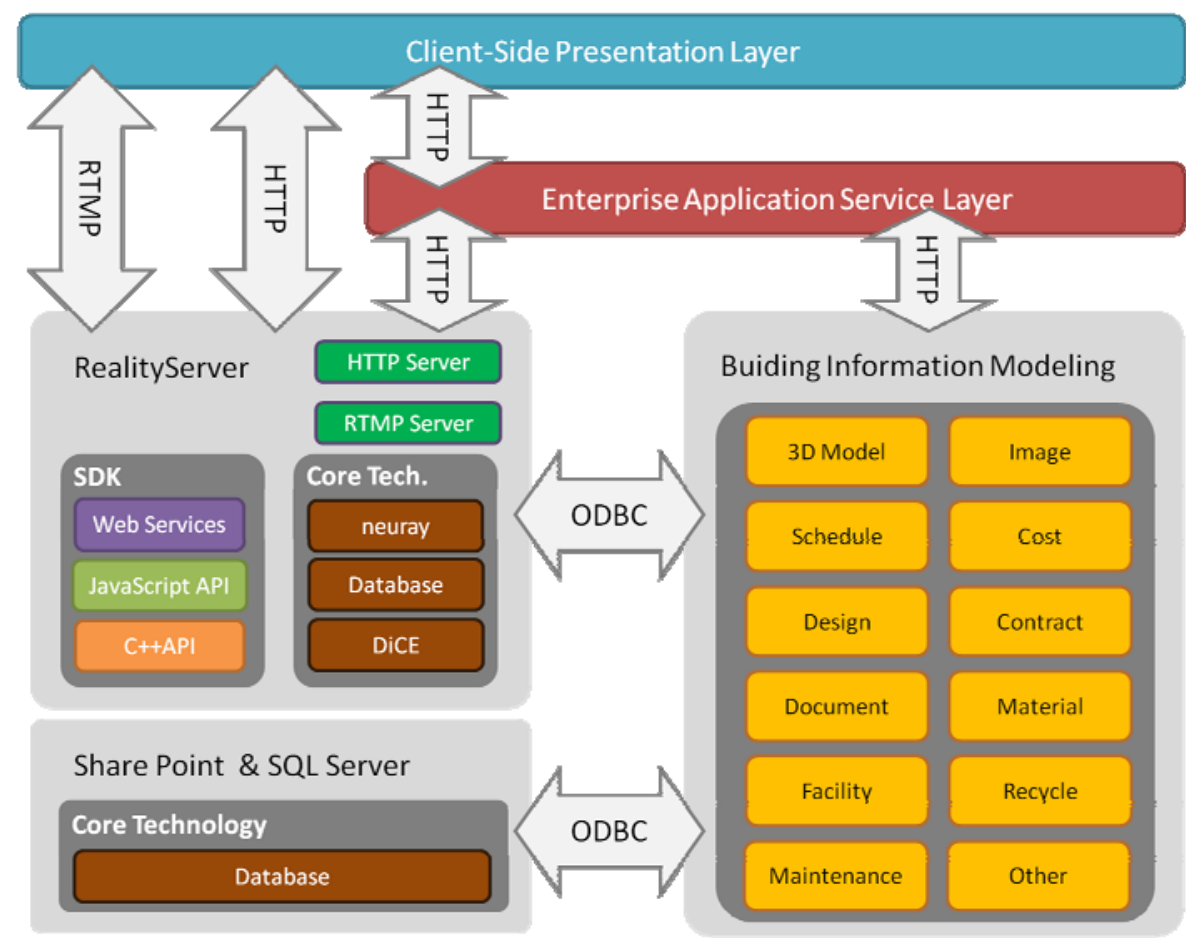

Figure 3. System Framework 


\subsection{GRAPHICAL USER INTERFACE}

This system provides a GUI to substitute the text-based UI of traditional engineering information management systems for efficient and effective facilitation of users' interaction with the system. Figure 4 illustrates the main GUI of the Cloud-BIM system.

\section{- 1D Visualization}

1D visualization of the system conveys information through text, attributes and tables to communicate basic project information. The frames A, C, F in the system are used to record and describe the project information in detail.

\section{- 2D Visualization}

This system can provide 2D visualization which presents information mainly through graphs, charts or maps. These systems provide signs (as shown in Table 2) and images to assist project managers in explaining project decisions and schedule rationale, and also enable clear evaluation of project goals as well as checking that project requirements are met (as indicated by Frames B and D).

Table 1. Color schema for sign of project progress

\begin{tabular}{|l|l|}
\hline Sign & \multicolumn{1}{|c|}{ Description } \\
\hline & Normal $(<1 \%)$ \\
\hline & Delay in schedule $(1 \% \sim 3 \%)$ \\
\hline & Serious delay in schedule $(>3 \%)$ \\
\hline
\end{tabular}

\section{- 3D Visualization}

This system can use 3D models to present the actual status and analyze space conflicts. Project goals and processes can be explained and evaluated even more accurately than through simple static 3D visualization. Frame E shows the graphical visualization of 3D models, which provides 3D manipulation functions such as fly, walk and rotate.

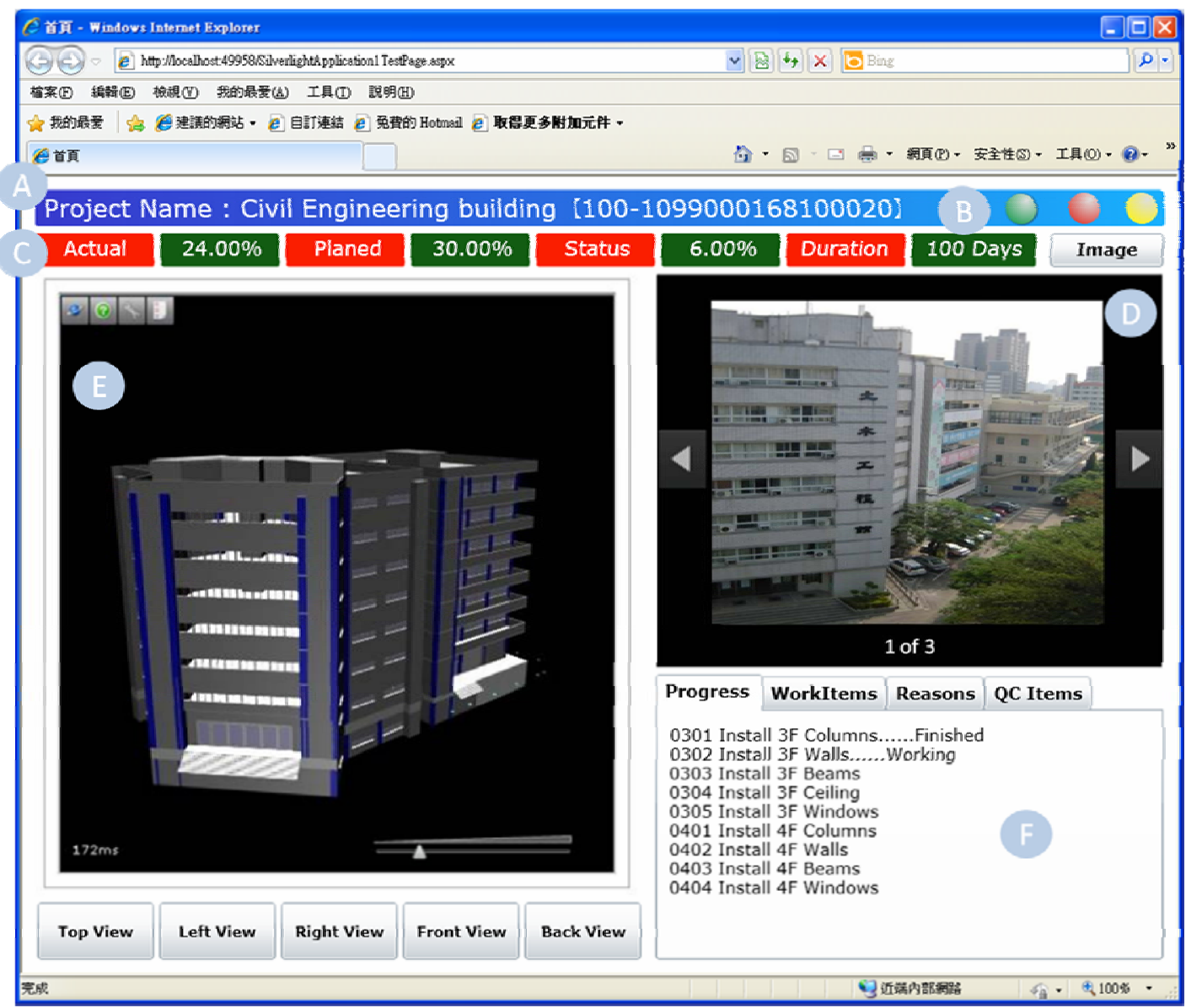

Figure 4. Graphical User Interface 


\section{CONCLUSION}

A growing number of case studies have shown the benefits of using BIM technology for building models. In the construction industry, the current trend is toward applying Building Information Modeling (BIM) in order to efficiently integrate and manage engineering information. Building models and BIM technology are set to become the standard method and technology for information representation in the foreseeable future [1]. However, as more people use BIM systems, there will be increasing emphases on their usability. Usability is a qualitative attribute that assesses UIs ease of use. This research has organized these GUI design rules and principles into what we consider the five most important UI design principles for engineering applications: Visibility, Feedback, Ease of remembering, Mapping and Consistency. The Cloud-BIM system was developed according to these five UI design principles. There are four major characteristics of the Cloud-BIM system: (1) Application of cloud-computing technology in the BIM system enables users to visualize and manipulate BIM information anytime and anywhere. (2) Following UI design principles to develop an effective and efficient GUI can facilitate communication and distribution of information between related participants, such as construction companies, building owners and architectural companies, for effective and efficient project management. (3) Providing a GUI to substitute the traditional text-based UI makes the system much easier to use. (4) By representing the project information visually, the project manager can quickly grasp the current status of an ongoing project. For the characteristics mentioned above, the Cloud-BIM system can not only visualize and manipulate BIM models; it can also effectively assist in controlling and monitoring the engineering project.

\section{REFERENCES}

[1] Chuck Eastman, Paul Teicholz, Rafael Sacks and Kathleen Liston, BIM Handbook: A Guide to Building Information Modeling for Owners, Managers, Designers, Engineers and Contractors, Wiley, 504 Pages, 2008.

[2] Michael Miller, Cloud Computing: Web-Based Applications That Change the Way You Work and Collaborate Online, Que, 312 Pages, 2008

[3] D.G. Treicher, Are You Missing the Boat in Training Aid?, Film and AV Communication 1 (1967) 14-16.

[4] RealityServer, RealityServer Technical Overview, mental images GmbH, Berlin, Germany, 2009.

[5] RealityServer, RealityServer 3.0 White Paper, mental images GmbH, Berlin, Germany, 2009.

[6] Donald A. Norman, The Design of Everyday Things, Basic Books, 272 Pages, 2002.

[7] Ben Shneiderman, Catherine Plaisant, Maxine Cohen and Steven Jacobs, Designing the User Interface: Strategies for Effective Human-Computer Interaction, 5th Edition, Addison Wesley, 624 pages, 2009.

[8] Jenifer Tidwell, Designing Interfaces, 2nd Edition, O'Reilly Media, 576 Pages, 2010.

[9] Susan Fowler and Victor Stanwick, Web Application Design Handbook: Best Practices for Web-Based Software, Morgan Kaufmann, 658 Pages, 2004.

[10] Jakob Nielsen and Hoa Loranger, Prioritizing Web Usability, New Riders Press, 432 Pages, 2006.

[11] International Organization for Standardization, Ergonomics of human-system interaction - Part 171: Guidance on software accessibility, ISO 9241171:2008, Retrieved March 25, 2011 from http://www.iso.org/iso/catalogue_detail.htm?csnumber $=39080$, 2008 . 